CAHIERS DE

NARRATOLOGIE
Cahiers de Narratologie

Analyse et théorie narratives

$33 \mid 2018$

L'Art du roman chez Umberto Eco

\title{
La presse entre le vrai et le faux dans Numéro Zéro d'Umberto Eco
}

\section{Tariq Oukhadda}

\section{(2) OpenEdition \\ Journals}

\section{Electronic version}

URL: http://journals.openedition.org/narratologie/8154

DOI: 10.4000/narratologie.8154

ISSN: 1765-307X

Publisher

LIRCES

\section{Electronic reference}

Tariq Oukhadda, «La presse entre le vrai et le faux dans Numéro Zéro d'Umberto Eco », Cahiers de Narratologie [Online], 33 | 2018, Online since 23 July 2018, connection on 14 November 2019. URL http://journals.openedition.org/narratologie/8154 ; DOI : 10.4000/narratologie.8154

This text was automatically generated on 14 November 2019.

Article L.111-1 du Code de la propriété intellectuelle. 


\title{
La presse entre le vrai et le faux dans Numéro Zéro d'Umberto Eco
}

\author{
Tariq Oukhadda
}

1 Umberto Eco accorde un grand intérêt à la question du faux. C'est une thématique qui lui est chère et qu'il a fini par installer comme horizon d'attente à la lecture de chacun de ses livres. L'univers créé par Eco mêle mensonge et vérité, ce n'est pas par cynisme ou nihilisme, mais pour montrer comment chacune des deux catégories arrive à produire du sens et à influencer les hommes et l'histoire. Numéro Zéro en est une parfaite illustration, c'est un roman qui traite d'une problématique d'actualité, à savoir les Fake News et la presse dans son rapport au lecteur et à la sphère politique. Il s'agira lors de notre analyse de montrer quels ressorts romanesques Eco mobilise-t-il pour produire un discours sur la presse écrite italienne? Nous prêterons à cet effet intérêt aux commentaires qui reflètent la subjectivité du narrateur, au point de vue et à l'ordre de la narration, aux personnages et au topos littéraire du complot.

2 Numéro Zéro raconte l'histoire d'un journal qui ne verra jamais le jour. Domani, le journal en question est dirigé par une équipe de "ratés" pour la plupart en reconversion professionnelle. Le rédacteur en chef, Simei, dirige le journal à la demande du Commandeur Vimercate pour de très sombres et tortueuses raisons. Pour intégrer les cercles bien fréquentés des finances et des banques, Vimercate charge Simei de produire une série de douze numéros pilotes, sans l'informer de sa véritable intention. Toutefois, Simei devine de lui-même que Domani n'est qu'une grande entreprise de manipulation. Une telle conclusion le pousse à mener un double jeu en faisant appel à Colonna, un nègre littéraire. Au début, ce dernier n'est pas du tout attiré par le monde de la presse, mais va s'avérer par la suite un très bon formateur pour les six rédacteurs choisis. Il connaît mieux le milieu universitaire, auquel il consacre plusieurs scènes dans sa narration. Il souligne l'hypocrisie qui y règne, parfois avec un humour sarcastique, et aussi pour montrer que le soi-disant pouvoir des maittres vient de quelques tours de passe-passe savamment dosés : «[...] l'orateur avançait entouré des professeurs titulaires, suivi à un mètre des chargés de cours, suivi à deux mètres des assistants et, à une distance raisonnable, des étudiants les plus téméraires »1. 
3 Quand Simei met Colonna dans la confidence et le charge d'écrire un livre, il lui demande de raconter le contraire de ce qui va se passer durant l'année de rodage de Domani. En jouant sur les deux tableaux, Simei espère tirer profit à la fois de sa position dans le journal et du livre qui paraitra par la suite et qui fera passer ses efforts et ceux de ses rédacteurs pour une noble quête de vérité.

$4 \quad$ Sur fond de complot et de suspicions incessantes, Eco brode une intrigue qui se déroule en Italie, dans le Milan des années 90. Dès les premières lignes, Numéro Zéro plonge le lecteur dans une intrigue qui ne cesse de se ramifier tout au long de la narration. D'emblée, Eco joue du doute et du mystère. Son lecteur devient un homo-suspicator en puissance, qui, au fil de la lecture, voit sa recherche de la vérité et de l'information se transformer en une obsession grandissante. Pour créer un tel effet, le premier chapitre du livre constitue une entrée en matière in medias res. Cette forme particulière d'incipit a pour fonction d'accrocher le lecteur et d'attiser sa curiosité. De ce fait, le lecteur entre de plain-pied dans l'intrigue. Pour plonger le lecteur dans cet univers de doutes et de questionnements, l'auteur fournit des détails disparates et parsème le texte de différentes allusions. Le lecteur se demande sans cesse : Qui est qui ? Qui fait quoi ? Qui cherche qui ? Qui veut tuer qui et pourquoi?

5 Colonna est le narrateur, mais aussi le personnage principal du roman. C'est à travers son point de vue que le lecteur prend part aux évènements. Étant le confident de Simei et des deux rédacteurs Braggadocio et Maia, Colonna permet au lecteur d'occuper une place de choix et l'invite à partager ses doutes et ses certitudes. En effet, son rôle est essentiel dans le déroulement des événements. C'est autour de lui que pivote toute l'histoire: il procède au briefing des rédacteurs, recueille les propos de Simei pour l'écriture du livre (Domani, Ieri) et prête une oreille attentive aux théories rocambolesques de Braggadocio.

6 Braggadocio est l'un des six rédacteurs de Domani. Il est passionné par les conspirations et les complots. Toutefois, ses thèses sur les juifs gazés par les Allemands, la guerre du Golfe et l'expédition américaine vers la lune finissent par convaincre Colonna que ce dernier est un mythomane. Braggadocio doute de tout et baigne dans une atmosphère où le complot prend une place principale. En effet, il affirme que "Nous vivons dans le mensonge et, si tu sais qu'on te ment, tu dois vivre dans le soupçon. Moi j'ai des soupçons, j'ai toujours des soupçons $»^{2} C^{\prime}$ est un de ses soupçons qui va déclencher un scandale et conduire à sa mort. Braggadocio incarne la sémiosis hermétique dans sa surinterprétation de tous les éléments qui l'entourent et dans son excès de soupçon parfois consciemment, parfois sans même s'en rendre compte.

7 Braggadocio n'est pas le seul à être porté sur le soupçon, Colonna l'est aussi. Tout commence dans son appartement. Quelqu'un a fermé l'arrivée d'eau. Sans doute eux. Cet incident mineur le transforme en une sorte de Sherlock Holmes, perdu entre le vrai et le faux. Il construit tout un scénario de cette prétendue visite, sans même songer à l'effet que peut avoir sur lui le Stilnox qu'il se souvient avoir pris la veille. Il décrète toutefois sa vulnérabilité et s'estime sans défense devant leur détermination. Face à cette dernière, il oppose une certaine lucidité par laquelle il explore toutes les répercussions possibles d'une action dont il n'a pas la certitude qu'elle ait eue lieu:

«Mes livres sont disposés dans leur désordre normal, les services secrets de la moitié du monde pourraient les avoir feuilletés page à page, que je ne m'en apercevrais pas. Il est inutile que je regarde dans les tiroirs ou que j'ouvre l'armoire de l'entrée. S'ils voulaient découvrir quelque chose, il ne leur restait qu'à fouiller dans l'ordinateur ${ }^{3}{ }^{3}$ 
Colonna se présume malgré tout chanceux et estime que détermination ne rime pas forcément avec intelligence : "Connards. S'ils étaient futés, ils ne seraient pas condamnés à faire un aussi sale métier. ${ }^{4}$ L'information qu'ils cherchent n'est ni dans son ordinateur ni dans ses livres ou ses notes. Elle est sur une disquette et il s'assigne de suite la mission de la protéger, convaincu qu'ils vont revenir à la charge et ne rien abandonner. Ces indications et menus détails dressent au fil des pages le portrait-type du personnage paranoïaque, adepte de la théorie du complot et qui cherche coûte que coûte à faire signifier l'insignifiant. C'était peut-être lui qui avait fermé l'arrivée d'eau. Il se peut aussi que personne n'ait visité son appartement. À travers le monologue de Colonna, Eco mène le lecteur pour ainsi dire en ballade. Ce labyrinthe d'inférences et de syllogismes pervers et tordus est un jeu avec le lecteur, mais cache quelque chose de sérieux, une idée très chère à Eco, celle de la fragilité de nos catégories et de nos raisonnements où parfois on ne peut plus déterminer ni savoir clairement ce qui est vrai et ce qui est faux.

9 La rétrologie complotiste à laquelle croit Braggadocio va se retrouver à l'épreuve de la presse qui doit la vérifier, la sérier et la mettre en discours. C'est le travail légué à Colonna après la mort de Braggadocio. Il va user, avec l'aide de Maia, du pouvoir de la presse et de l'investigation pour déterminer l'authenticité des thèses de son collègue. En effet, la presse écrite exerce un contre-pouvoir dont Eco a restitué la configuration et le fonctionnement. Le pouvoir à contrecarrer ici est celui des évènements du passé qui imposent: "un corps permanent, auquel on a l'habitude d'obéir, qui a les moyens de contraindre, et qui est soutenu par l'opinion qu'on a de sa force, la croyance dans son droit de commander (sa légitimité), et l'espoir qu'on met dans sa bienfaisance. $»^{5}$.

10 C'est ce lourd poids historique qu'Eco tente de repenser à l'aune de cette critique des médias insérée dans un moule romanesque. Une telle entreprise s'est accompagnée d'une critique acerbe, mais parfois exagérée. Eco le dit lui-même lors d'un entretien ${ }^{6}$ : "J'ai donné une représentation peut-être extrême et grotesque de la vie d'un journal.» En écrivant un "condensé de faux et de vices du journalisme », Eco montre ainsi subtilement comment le faux s'insinue dans le discours des journalistes à leur insu, parce que les mots les poussent en quelque sorte à le faire et parce qu'ils sont manipulés. Il évoque par la même le concept d'Agenda-setting, terme qui désigne la hiérarchisation des nouvelles de manière à donner ou à imposer une certaine interprétation. C'est aussi une démonstration qui révèle toute l'aisance avec laquelle un quotidien est en mesure de créer un univers de toutes pièces, en jouant seulement de la disposition et du choix des nouvelles. En effet, «Les journaux ne sont pas faits pour diffuser, mais pour couvrir les nouvelles $»^{7}$

11 La presse est soumise aussi à la logique du marché et du post-capitalisme où il s'agit d'optimiser au maximum et de faire gagner plus dans sa capacité à générer plusieurs versions d'une même vérité. Au fait, "Ce ne sont pas les informations qui font le journal, mais le journal qui fait l'information. Et savoir rassembler quatre nouvelles différentes signifie en proposer au lecteur une cinquième. $»^{8}$. L'information est une construction et l'effet qu'elle produit dépend fortement de la ligne éditoriale. Celle de Domani est des plus claires : c'est un journal dédié à la recherche de la vérité, la quête des vérités qui dérangent aux dépens de celles qui apaisent, puisque le but de cette recherche est la mise en place d'un pouvoir qui servirait à un chantage éhonté.

12 Ce que construit la presse n'est pas un mensonge, mais un certain jeu avec la vérité. La presse revêt donc une dimension toute autre en faisant de son principal lieu 
d'expression, le journal, un moyen essentiel pour le monde des affaires et de la politique. Pour ce faire, les médias construisent des pseudo-réalités, du faux aux apparences véridiques. L'exécution déjouée du Duce et sa fuite en Argentine sont autant de pseudo-évènements qui font du roman une sorte de journal par correspondance, destiné à des abonnés férus de sensations et de complot.

Jadis le rôle de la presse était d'informer et de former une opinion sur les choses de ce monde. Aujourd'hui l'opinion n'est pas le but, mais c'est la consommation immédiate de petites opinions instantanées, destinées à être consommées sur place et oubliées dans les heures qui suivent pour laisser la place à celles qu'on est déjà en train de boucler.

14 On serait tenté de voir cela comme l'expression d'une certaine éthique, du moins d'un certain engagement de la part d'Eco. D'abord parce qu'on constate qu'Eco développe une critique du fonctionnement de la presse écrite. Ensuite, parce qu'en expliquant ce qu'un journal ne doit pas faire, il exprimerait une volonté de dépasser les difficultés actuelles afin de redonner à la presse les moyens de remplir de nouveau ses fonctions sociales. De ce fait, les personnages tiennent des propos qui s'adressent tantôt à ceux qui font la presse tantôt à ceux qui la lisent. Eco livrerait au lecteur un manuel riche en recommandations, conseils et mises en garde. Par exemple, Colonna offrait aux six rédacteurs de Domani plusieurs conseils. Chaque choix éditorial est dicté par la représentation que le journal se fait de son lectorat. Pour Simei, les éléments de langage que son équipe utilise sont tributaires de la culture des lecteurs. Il conseille : « Ne suscitons pas de complexes d'infériorité dans le public. "9. De même, Simei insiste à plusieurs reprises sur l'uniformité du style du journal qui ne doit pas dérouter le lecteur. Un journal prend également en considération le statut social de ses lecteurs. $\mathrm{Ne}$ lui sont pas également indifférents les goûts du lecteur. Concernant un sujet sur les téléphones portables, Simei conseille: "Pour nos lecteurs, qui en majorité n'en possèdent pas, le sujet n'a aucun intérêt, et pour ceux qui en ont un, peu nombreux, ça ne leur fait ni chaud ni froid, pire : ils nous prendraient pour des snobs, des radicaux chics. $»^{10}$

15 Certes la presse a une responsabilité sociale. Elle doit être en mesure de proposer un contenu uniforme répondant aux attentes des lecteurs. Sa fonction principale est d'informer, mais aussi de démêler le faux du vrai. Son rôle est donc de revisiter l'Histoire et de jeter un regard critique sur les événements du passé. Mais toute la bonne volonté du monde ne peut rien contre la machine culturelle et historique qui broie et intègre tout. Eco écrit dans La guerre du faux que « le faux est "historique" et comme tel, il est déjà revêtu d'authenticité.» ${ }^{11}$. C'est cette évidence que veut questionner Umberto Eco en ouvrant les placards fermés de l'histoire de l'Italie de l'après-guerre. Une hypothèse des plus intéressantes à relever dans ce sens supposerait que la mort du Duce ne serait qu'un prétexte à la production d'un discours sur la presse. Le mensonge tout comme la vérité fait partie de l'histoire. Eco aborde cela avec un calme et une sérénité intrigante pour le lecteur naïf, mais très compréhensible pour le lecteur critique habitué, celle de quelqu'un qui avait à maintes reprises réfléchi et débattu de façon sérieuse sur la vérité et le mensonge. Celle de quelqu'un qui a longtemps réfléchi sur les mécanismes de la fable et sur comment chaque fable construit son propre lecteur, puisque le plus important, ce n'est pas de raconter la vérité ou le mensonge, du moment que raconter est toujours quelque part un mensonge même lorsque cela concerne les faits les plus avérés. 
16 Eco avait consacré de nombreuses longues années à réfléchir sur le vrai et le faux au point de leur consacrer une typologie détaillée dans Les Limites de l'interprétation. Et il est plus que judicieux de rappeler ici ses conclusions pour donner à voir dans quel état d'esprit Numéro Zéro a été écrit. Le répertoire des cas de contrefaçon ou de ressemblance n'est possible qu'en partant de propositions primitives sur le type de similarité, de ressemblance ou d'iconisme. En ce qui concerne la contrefaçon en général, une sémiotique du mensonge permet de considérer qu'on n'a pas affaire directement à des mensonges. Nous avons affaire plutôt à la possibilité de prendre un objet pour un autre, avec lequel il partage certains traits en commun. En distinguant contrefaçon radicale et contrefaçon ex nihilo, Eco parvient à mettre en évidence l'aspect foncièrement pragmatique de la contrefaçon: «on a contrefaçon lorsqu'un objet est produit - et, une fois produit, utilisé ou exposé - avec l'intention de faire croire qu'il est indiscernablement identique à un autre objet unique » (Eco (1990) 1992 : 183)

17 L'accent mis sur la dimension pragmatique du problème de la contrefaçon (intention de tromper) permet cette autre définition du faux que l'auteur formule ainsi : «Quelque chose n'est donc pas un faux à cause de ses propriétés internes, mais en vertu d'une identité prétendue. Ainsi, les contrefaçons posent avant tout un problème pragmatique. » (Eco (1990) $1992: 184)$

18 Ceci déplace l'analyse du côté du vrai et de l'authentique. Autrement dit, le problème crucial d'une sémiotique du faux n'est pas de répertorier les erreurs du prétendant, mais de dire sur quoi le juge s'est appuyé pour démontrer que l'objet contrefait est authentique. Comme on peut le remarquer, le problème est circulaire. Eco adopte ici une position rigoureusement nominaliste où une chose est vraie de telle sorte qu'elle n'est pas fausse.

19 On peut être tenté de prendre les positions d'Eco pour un certain type d'engagement, voire pour une sorte de marxisme, cela est défendable à bien des égards, surtout chez le sémioticien du Trattato di semiotica generale, qui a évolué sous d'autres formes par la suite, mais encore une fois, ce serait oublier la conviction profondément sémiotique et le souci permanent du romancier doublé du sémioticien qui veut plus attirer l'attention sur le mécanisme pragmatique du mensonge et du faux que sur son économie politique.

\section{BIBLIOGRAPHY}

De Jouvenel, Bertrand, Du pouvoir, Histoire naturelle de sa croissance, Hachette, coll. Pluriel, Paris, 1972.

Eco, Umberto, La guerre du faux, Grasset, Paris, 1985.

Eco, Umberto, Numéro Zéro, Grasset, Paris, 2015. 


\section{NOTES}

1. Eco, Umberto, Numéro Zéro, op. cit., pp.18-19.

2. Ibid., p.45.

3. Ibid., pp.12-13.

4. Ibid., p.13.

5. De Jouvenel, Bertrand, Du pouvoir, Histoire naturelle de sa croissance, Hachette, coll. Pluriel, Paris, 1972, p. 54.

6. Entretien du 25-05-2015, FNAC des Ternes, Paris.

7. Eco, Umberto, Numéro Zéro, op. cit., p.174.

8. Ibid., p.59.

9. Eco, Umberto, Numéro Zéro, op. cit., p.99.

10. Ibid., p.98.

11. Eco, Umberto, La guerre du faux, Grasset, Paris, 1985, p.33

\section{ABSTRACTS}

This article sheds light on Umberto Eco's latest novel entitled Zero issue which focuses on a specific problematic, notably that of the relationship between media and fake news. Such a relationship is considered very complex because it is closely linked to many economic, political, but also semiotic issues. Umberto Eco renews once again with the theme, dear to him, which is that of the power of liying. This time, what is pointed out is the drifting of information when it is seized by a triumphant capitalism and by a society more and more liquid, without memory, without history and without readers.

Cet article met en lumière le dernier roman d'Umberto Eco intitulé Numéro Zéro qui se penche sur la problématique spécifique de la relation entre les médias et les fake news. Une telle relation est considérée comme très complexe car étroitement liée à de nombreuses questions économiques, politiques mais aussi sémiotiques. Umberto Eco renoue une fois de plus avec un thème qui lui est cher, celui du pouvoir du mensonge. Cet article souligne la dérive de l'information saisie par un capitalisme triomphant et par une société de plus en plus liquide, sans mémoire, sans histoire et sans lecteurs.

\section{INDEX}

Keywords: fake news, misinformation, zero issue, manipulation, lie

Mots-clés: fake news, désinformation, Numéro zéro, manipulation, mensonge

\section{AUTHOR}

\section{TARIQ OUKHADDA}

École doctorale, Faculté des Lettres de Meknès 